\title{
A Comparison of Full-Rate Full-Diversity $2 \times 2$ Space-Time Codes for WiMAX Systems
}

\author{
Serdar Sezginer $^{(1)}$, Hikmet Sari ${ }^{(1),(2)}$ and Ezio Biglieri ${ }^{(1)}$ \\ (1) Sequans Communications, Bat. Citicenter, 19 Le Parvis, 92073 Paris La Défense, France \\ ${ }^{(2)}$ Supélec, Plateau de Moulon, 1-3 Rue Joliot Curie, 91190 Gif sur Yvette, France
}

\begin{abstract}
Multiple-input multiple-output (MIMO) techniques have become an essential part of broadband wireless communications systems. For example, the recently developed IEEE 802.16e specifications for broadband wireless access include three MIMO profiles employing $2 \times 2$ space-time codes (STCs), and two of these MIMO schemes are mandatory on the downlink of Mobile WiMAX systems. One of these has full rate, and the other has full diversity, but neither of them has both of the desired features. The third profile, namely, Matrix $\mathrm{C}$, which is not mandatory, is both a full rate and a full diversity code, but it has a high decoder complexity. Recently, the attention was turned to the decoder complexity issue and including this in the design criteria, several full-rate STCs were proposed as alternatives to Matrix C. In this paper, we review these different alternatives and compare them to Matrix $\mathrm{C}$ in terms of performances and the corresponding receiver complexities.
\end{abstract}

Keywords-Multiple-input multiple-output (MIMO), spacetime codes (STCs), WiMAX systems.

\section{INTRODUCTION}

Multiple-input multiple-output (MIMO) techniques based on using multiple antennas at transmitter and receiver can provide spatial diversity, multiplexing gain, interference suppression, and make various tradeoffs between them. These techniques have been incorporated in all of the recently developed wireless communications system specifications and have become an indispensable part of the IEEE 802.16e-2005 standard [1] for mobile broadband wireless access systems.

From the MIMO schemes included in the IEEE 802.16e specifications, the WiMAX Forum has specified two mandatory profiles for use on the downlink. One of them is based on the space-time code (STC) proposed by Alamouti for transmit diversity [2]. This code achieves a diversity order that is equal to twice the number of antennas at the receiver, but it is only half-rate, because it only transmits two symbols using two time slots and two transmit antennas. (In this paper, the rate is defined as the number of transmitted symbols per antenna use. The Alamouti code is of course full rate if the rate is defined as the number of transmitted symbols per channel use). The other profile is spatial multiplexing (SM), which uses two transmit antennas to transmit two independent data streams. This scheme is full-rate, but it does not benefit from any diversity gain at the transmitter, and at best, it provides a diversity order equal to the number of receive antennas.

For future evolutions of the WiMAX standard, it is highly desirable to include a new code combining the respective advantages of the Alamouti code (Matrix A) and the SM (Matrix B) while avoiding their drawbacks. Such a code actually exists in the IEEE 802.16e-2005 specifications (where it is referred to as Matrix C). The Matrix $\mathrm{C}$ is a variant of the Golden code [3] (see also [4] and [5] for other variants), which is known to be one of the best $2 \times 2$ STCs achieving the diversity-multiplexing frontier [4]. But the problem of this code is its detection complexity, which grows as the fourthpower of the signal constellation size, and this makes it impractical for low-cost wireless user terminals.

Recently, motivated by the orthogonality of the Alamouti scheme, new full-rate full-diversity (FR-FD) $2 \times 2$ STCs were proposed independently in [6][7][12]. These codes achieve the diversity-multiplexing frontier, while their optimum detection complexity (using exhaustive search) grows at most quadratically with the size of the signal constellation (see, e.g., [6] for more detail).

In this paper, we present a unified comparison between Matrix C and the STC presented in [6]. First, in Section II, we briefly discuss the design criteria for STCs. Sections III is devoted to the comparison between Matrix $\mathrm{C}$ and the recently proposed codes. Then, in Section IV, we explain the sphere decoder (SD) and the corresponding reduced SD. Finally, we present some numerical comparisons in Section $\mathrm{V}$ and give our conclusions in Section VI.

Notation: Matrices (resp. column vectors) are set in boldface capital (resp. lower case) letters. $a_{k l}$ denotes the entry of matrix $\mathbf{A}$ at its $k$ th row and $l$ th column, and $b_{k}$ denotes the $k$ th element of the column vector $\mathbf{b}$. The operators $(\cdot)^{*},(\cdot)^{T}$, and $(\cdot)^{H}$ stand for complex conjugate, transpose, and conjugate transpose, respectively. $\|\cdot\|^{2}$ denotes Frobenius norm of the enclosed vector.

\section{STC DESIGN CRITERIA}

\section{A. Pairwise Error Probability Analysis}

Now, we will briefly discuss the most common design criteria for STCs. We consider that the transmitter does not have any channel state information while the receiver knows the channel perfectly. For $2 \times 2$ MIMO transmission, we write

$$
\mathbf{Y}=\mathbf{H X}+\mathbf{Z},
$$

where $\mathbf{H}$ is the $2 \times 2$ channel matrix with the entries $h_{k l}$ of complex channel gains, $\mathbf{X}$ is the $2 \times 2$ codeword matrix 


$$
\mathbf{X}=\left[\begin{array}{ll}
x_{11} & x_{12} \\
x_{21} & x_{22}
\end{array}\right]
$$

whose elements take values from the codebook $X, \mathbf{Y}$ includes the received signal samples and $\mathbf{Z}$ denotes the matrix of additive circularly symmetric complex Gaussian noise samples with spectral density $N_{0}$. The recently proposed STC schemes mainly rely on the analysis of pairwise error probability (PEP) $P(\mathbf{X} \rightarrow \hat{\mathbf{X}})$ which is the probability that $\hat{\mathbf{X}}$ is detected while $\mathbf{X}$ is transmitted. At high signal-to-noise ratio (SNR) values, the union-Chernoff bound analysis of the PEP leads to the following design criteria.

1) Rank Criterion [8]: For the STC given in (2), the diversity gain, defined as

$$
d(X)=\min _{\substack{\mathbf{X}, \hat{\mathbf{X}} \in X \\ \mathbf{X} \neq \hat{\mathbf{X}}}} \operatorname{rank}(\mathbf{X}-\hat{\mathbf{X}}),
$$

should be maximized. If $(\mathbf{X}-\hat{\mathbf{X}})$ is full rank for all codeword pairs, then the code is said to have full diversity.

2) Determinant Criterion [10]: After ensuring full-diversity, we should maximize the coding gain which can be defined for a $2 \times 2$ STC as

$$
\delta=\min _{\substack{\mathbf{X}, \mathbf{X} \in X \\ \mathbf{X} \neq \mathbf{\mathbf { X }}}}|\operatorname{det}(\mathbf{X}-\hat{\mathbf{X}})|^{2} .
$$

In order to obtain the best achievable performance, the coding gain should be maximized for a given average transmit power. It should be noted that, for high SNR values, the most important parameter is the diversity gain which dominates the steepness of the bit-error rate (BER) curve. Afterwards, it is the coding gain which should be maximized. The STCs, presented in the sequel, are examples of such full-diversity schemes which have large coding gain. Other design criteria can be added. Among them, we mention here the requirement that the constellation has cubic shaping (see below for a discussion).

\section{B. Detection Complexity}

In the design of STCs another important criterion is the decoding complexity. This is highly crucial especially for mobile applications. The Matrix C (or equivalently the Golden code) is the best-known full-rate $2 \times 2$ STC which satisfies the rank criterion with a high coding gain. However, optimum detection has a high computational complexity. Therefore, other FR-FD STCs with lower optimum decoding complexity should be included as alternatives to Matrix C. The results available in the literature suggest that there is an intrinsic tradeoff between error performance and detection complexity. But, theoretical tradeoff limits have not been exhibited yet.

\section{FR-FD $2 \times 2$ STCS FOR WIMAX SYSTEMS}

Matrix $C$ has been included in the IEEE 802.16-2005 specifications for the enhancement of the performance of Matrix A and B while providing full-rate and full-diversity with a higher coding gain. Particularly, for a group of 4 symbols $\left(s_{1}, s_{2}, s_{3}, s_{4}\right)$, the transmission matrix is given by

$$
\mathbf{X}_{C}=\frac{1}{\sqrt{1+r^{2}}}\left[\begin{array}{cc}
s_{1}+j r s_{4} & r s_{2}+s_{3} \\
s_{2}-r s_{3} & j r s_{1}+s_{4}
\end{array}\right]
$$

where $r=(-1+\sqrt{5}) / 2$ and $j=\sqrt{-1}$.

This code leads to a spatial diversity of order 4 for 2 receiver antennas and achieves substantially better performance than the SM code (Matrix B) whose spatial diversity is limited to 2 (for this number of receive antennas). Moreover, $\mathbf{X}_{C}$ results in the same bit error probability as the Golden code (and the other variants proposed in [4] and [5]). More specifically, $\mathbf{X}_{C}$ results in a coding gain of $16 / 5$ which is the largest coding gain obtained so far. $\mathbf{X}_{C}$ differs from the Golden code and its variants in using a higher order extension field (see, e.g., [9] for related definitions). From the implementation point of view, the only difference is that the construction of $\mathbf{X}_{C}$ requires a smaller number of multiplications compared to the Golden code. However, as explained above, the problem of this code is its inherent detection complexity.

In particular, the optimum receiver evaluates the ML function for all symbol quadruplets $\left(s_{1}, s_{2}, s_{3}, s_{4}\right)$ and selects the one which maximizes this ML function. The ML function evaluated for $\left(s_{1}, s_{2}, s_{3}, s_{4}\right)$ is actually the squared Euclidean distance between the received noisy signal and the noiseless signal corresponding to that quadruplet, and can be expressed as the squared Frobenius norm

$$
D\left(s_{1}, s_{2}, s_{3}, s_{4}\right)=\|\mathbf{Y}-\mathbf{H X}\|^{2} .
$$

For a signal constellation with $M$ points, this receiver involves the computation of $M^{4}$ Euclidean distances and selects the symbol quadruplet minimizing this distance. The optimum receiver complexity is, therefore, proportional to $16^{4}=65.536$ for a 16-QAM signal constellation, and to $64^{4}=16.777 .216$ for a 64-QAM signal constellation. Of course, this is prohibitive in practical applications. Therefore, one resorts to suboptimum receivers which may degrade the performance severely. One possible solution is to use SD whose performance and complexity are upper bounded by those of ML detection based on exhaustive search. The major issues in the implementation of SD are choosing the initial radius and the order in which the symbols are examined. These two issues can dramatically improve or degrade the complexity of SD. In fact, even the SD would require a high number of computations for satisfactory detection performance. This requires the use of new STCs which have close performance to that of $\mathbf{X}_{C}$ with lower detection complexity. It is also worth noting that, from a practical point of view, SD suffers from the fact that its throughput is variable.

Now, we turn our attention to the recently proposed FR-FD $2 \times 2$ STC schemes which may be very strong candidates for future high-rate wireless transmission systems. They attempt to maximize both the diversity gain and the coding gain, while leading to an optimum detection of reduced complexity. More 
specifically, these schemes are FR-FD $2 \times 2$ STCs whose optimum receiver has a complexity that is only proportional to $M^{2}$ (see [6][7][11]-[13] for more detail). Thus, the number of Euclidean distance computations in the optimum detector is reduced to $16^{2}=256$ for a $16-\mathrm{QAM}$ signal constellation and to $64^{2}=4.096$ for a 64-QAM signal constellation. Comparing these numbers to those associated to Matrix $\mathrm{C}$, it becomes clear that these codes make the implementation of FR-FD $2 \times 2$ STCs with optimum receiver more realistic.

Such an STC first appears in [12] but its low decoding complexity property was only realized in [7] independent from our work in [6]. The STC presented in [7] is a combination of the original Alamouti scheme and a precoded scheme having also an Alamouti structure. In contrast, our STC has a symmetric structure since it directly combines two Alamouti schemes. As is shown in the sequel, this evenly distributes the transmitted energy for each symbol per channel use. Moreover, since they lead to the same detection complexity and almost the same performance [13], we prefer to present the simplest one in terms of construction, namely, the STC that we recently presented in [6]. In this code, the group of 4 symbols $\left(s_{1}, s_{2}, s_{3}, s_{4}\right)$ is transmitted as follows:

$$
\mathbf{X}_{\text {new }}=\left[\begin{array}{cc}
a s_{1}+b s_{3} & -c s_{2}^{*}-d s_{4}^{*} \\
a s_{2}+b s_{4} & c s_{1}^{*}+d s_{3}^{*}
\end{array}\right] .
$$

A careful look clearly shows that (7) is nothing but a simple linear combination of two Alamouti schemes. Here, $a, b, c$, and $d$ are complex-valued design parameters. They are chosen such that the resulting STC attains FR-FD transmission in a quasi-static Rayleigh fading channel. However, this task is infeasible especially for higher constellation sizes. In [6], the optimization of the parameters is simplified considering the desired average transmit power constraints. More specifically, we consider

$$
\begin{aligned}
& |a|^{2}+|b|^{2}=|c|^{2}+|d|^{2}=1 \\
& |a|^{2}+|c|^{2}=|b|^{2}+|d|^{2}=1
\end{aligned}
$$

as the transmit power constraints. The first condition ensures the transmission of equal average power at each symbol time, while the second condition ensures that equal average total power is transmitted for each symbol. A simple manipulation can show that the magnitudes of $a$ and $c$ should be equal for reduced complexity optimum detection. These equalities lead immediately to the fact that all the design parameters should have the same magnitude, i.e., $|a|=|b|=|c|=|d|=1 / \sqrt{2}$.

Now, without any loss of generality, we may set $a=c=1 / \sqrt{2}$. This decreases the number of unknown parameters without affecting the coding gain. Then, the remaining parameter pair $(b, d)$ can be optimized numerically leading to a full-diversity scheme with large coding gain. Such an optimization has been performed using QPSK signaling and resulted in a set of parameter pairs which gave a coding gain of 2 independent from QAM constellation size. In light of the design criteria given in Section II, this directly ensures that the obtained code will have full diversity for any QAM constellation size. Moreover, as shown in [4] and [14], such an STC with non-vanishing coding gain achieves the diversitymultiplexing frontier. In the numerical illustrations we provide the example of [6] in which we have $b=[(1-\sqrt{7})+j(1+\sqrt{7})] /(4 \sqrt{2})$ and $d=-j b$.

Both of the above mentioned STCs fall into the class of linear dispersion codes [15] which can be written in the form

$$
\mathbf{X}=\sum_{k=1}^{4}\left(s_{k, R} \mathbf{A}_{k}+j s_{k, I} \mathbf{B}_{k}\right),
$$

where $s_{k, R}$ and $s_{k, I}$ denote the real and imaginary parts of the symbol $s_{k}$, respectively, and $\mathbf{A}_{k}, \mathbf{B}_{k}, k=1, \ldots, 4$, are $2 \times 2$ complex-valued weight matrices of $\mathbf{X}$. The matrices $\mathbf{A}_{k}, \mathbf{B}_{k}$, $k=1, \ldots, 4$ have to be designed such that

$$
\sum_{k=1}^{4} \operatorname{tr}\left(\mathbf{A}_{k}^{H} \mathbf{A}_{k}+\mathbf{B}_{k}^{H} \mathbf{B}_{k}\right)=8
$$

in order to conserve the total average transmitted power, where $\operatorname{tr}(\cdot)$ denotes the trace of the enclosed matrix. With the constraint of equal average energy transmission for each symbol, (11) turns to

$$
\operatorname{tr}\left(\mathbf{A}_{k}^{H} \mathbf{A}_{k}+\mathbf{B}_{k}^{H} \mathbf{B}_{k}\right)=2 \text { for all } k=1, \ldots, 4 .
$$

It can be easily shown that the aforementioned STCs satisfy (12). Indeed, (12) is equivalent to the transmit power constraint (9) used in the design of $\mathbf{X}_{n e w}$. Furthermore, since the magnitudes of all the parameters are equal in $\mathbf{X}_{n e w}$, all the symbols will be transmitted with the same average power at each channel use. This property is unique to $\mathbf{X}_{n e w}$.

Now, in order to make a more detailed comparison, we use vector representation and introduce the following notation.

First, define the column vectors $\overline{\mathbf{x}}=\left[x_{11}, x_{21}, x_{12}, x_{22}\right]^{T}$, $\overline{\mathbf{y}}=\left[y_{11}, y_{21}, y_{12}, y_{22}\right]^{T}$ and $\overline{\mathbf{z}}=\left[z_{11}, z_{21}, z_{12}, z_{22}\right]^{T}$, which are obtained from stacking the columns of the matrices $\mathbf{X}, \mathbf{Y}$ and $\mathbf{Z}$, respectively, one after the other. Next, we define the corresponding real-valued column vector as

$$
\overline{\mathbf{x}}_{R}=\left[\operatorname{Re}\left\{x_{11}\right\}, \operatorname{Im}\left\{x_{11}\right\}, \operatorname{Re}\left\{x_{21}\right\}, \operatorname{Im}\left\{x_{21}\right\}, \ldots, \operatorname{Re}\left\{x_{22}\right\}, \operatorname{Im}\left\{x_{22}\right\}\right]^{T}
$$

It is known that any linear dispersion code in the form of (10) can be expressed as

$$
\overline{\mathbf{x}}_{R}=\mathbf{G} \overline{\mathbf{s}}_{R},
$$

where $\overline{\mathbf{s}}_{R}$ collects the real and imaginary parts of the symbols from the symbol vector $\mathbf{s}=\left[s_{1}, s_{2}, s_{3}, s_{4}\right]^{T}$ as in (13). Here, the matrix $\mathbf{G}$ is called the real generator matrix of the STC.

The Matrix $\mathrm{C}\left(\mathbf{X}_{C}\right)$ and the STC presented in [7] has the property that the generator matrix $\mathbf{G}$ satisfies

$$
\mathbf{G G}^{T}=\mathbf{G}^{T} \mathbf{G}=\mathbf{I}_{8},
$$

where $\mathbf{I}_{N}$ denotes the $N \times N$ identity matrix. Therefore, the properties of the input signal $\mathbf{s}$ is not changed and the resulting 
STC is said to have cubic shaping [3]. This also implies that the average power of the input symbol vector $\mathbf{s}$ remains unchanged whatever the structure of the signal. On the other hand, the property (15) is not satisfied with $\mathbf{X}_{\text {new }}$, hence, it does not have cubic shaping. To that end, in order to make a fair comparison between $\mathbf{X}_{C}$ and $\mathbf{X}_{\text {new }}$, we need to know the statistics of the input symbols. In fact, if the input symbols $s_{1}, s_{2}, s_{3}, s_{4}$ are either non-zero mean independent symbols or correlated symbols, then, the performance of $\mathbf{X}_{n e w}$ will deviate from that of $\mathbf{X}_{C}$. However, since quite powerful interleavers are used in all current system specifications, it is reasonable to assume that the data symbols at the input of the space-time encoder will be uncorrelated. Hence, the average performance of the system will not be affected by the absence of property in (15). Indeed, the average power is conserved when the input symbols have no correlation, i.e., we have

$$
E\left[\left\|\mathbf{G} \overline{\mathbf{s}}_{R}\right\|^{2}\right]=E\left[\overline{\mathbf{s}}_{R}^{T} \mathbf{G}^{T} \mathbf{G} \overline{\mathbf{s}}_{R}\right]=E\left[\left\|\overline{\mathbf{s}}_{R}\right\|^{2}\right]=E\left[\|\mathbf{s}\|^{2}\right],
$$

for both $\mathbf{X}_{C}$ and $\mathbf{X}_{\text {new }}$.

\section{DECODING OF FR-FD $2 \times 2$ STCS}

Now, we provide a comparison of the STCs in terms of decoding complexity. In [6], for the sake of simplicity, direct utilization of exhaustive search was described. Here, we will describe the SD and present the reduced complexity detector employing the SD.

\section{A. Sphere Decoding}

Utilizing the definition (13) for the column vectors $\overline{\mathbf{y}}$ and $\overline{\mathbf{z}}$, we can express the MIMO transmission in (1) as [16]

$$
\overline{\mathbf{y}}_{R}=\breve{\mathbf{H}} \mathbf{G} \overline{\mathbf{s}}_{R}+\overline{\mathbf{z}}_{R} .
$$

Here, $\breve{\mathbf{H}}$ is obtained from the channel matrix $\mathbf{H}$ as $\breve{\mathbf{H}}=(1 / 2) \mathbf{I}_{2} \otimes\left(\mathbf{H} \otimes \mathbf{E}+\mathbf{H}^{*} \otimes \mathbf{E}^{*}\right)$ where $\otimes$ stands for Kronecker product and $\mathbf{E}=\left[\begin{array}{cc}1 & j \\ -j & 1\end{array}\right]$. Then, the ML metric (6) can be rewritten as

$$
D\left(s_{1}, s_{2}, s_{3}, s_{4}\right)=\left\|\overline{\mathbf{y}}_{R}-\breve{\mathbf{H}} \mathbf{G} \overline{\mathbf{s}}_{R}\right\|^{2} .
$$

Minimization of (17) can be implemented using the SD algorithm [17] which is more computationally efficient than the exhaustive search in most cases. To this end, the matrix $\breve{\text { HG }}$ is first decomposed using $\mathbf{Q R}$ decomposition as $\breve{\mathbf{H}} \mathbf{G}=\mathbf{Q R}$, where $\mathbf{Q}$ is an $8 \times 8$ unitary matrix and $\mathbf{R}$ is an $8 \times 8$ upper triangular matrix. Multiplying (16) from left-hand side with $\mathbf{Q}^{H}$, we rewrite the input-output relation as

$$
\widetilde{\mathbf{y}}_{R}=\mathbf{Q}^{H} \overline{\mathbf{y}}_{R}=\mathbf{R} \overline{\mathbf{s}}_{R}+\mathbf{Q}^{H} \overline{\mathbf{z}}_{R} .
$$

Then, the SD finds

$$
\hat{\overline{\mathbf{s}}}_{R}=\underset{\overline{\mathbf{s}}_{R}}{\arg \min }\left\|\overline{\mathbf{y}}_{R}-\mathbf{R} \overline{\mathbf{s}}_{R}\right\|^{2} .
$$

The search procedure of this standard real SD should be performed by using a tree search with 8 levels. Now, using the special structures of its real generator matrix $\mathbf{G}$ and the upper triangular matrix $\mathbf{R}$, we will show that $\mathbf{X}_{\text {new }}$ lends itself to a reduced complexity implementation of the SD.

\section{B. Reduced-complexity detection}

Using the fact that the QR decomposition coincides with the Gram-Schmidt orthogonalization procedure applied to the columns of the matrix $\breve{\mathbf{H}}$ (see [13] for more detailed discussion), it can be shown that the upper-triangular matrix $\mathbf{R}$ appears to be

$$
\mathbf{R}=\left[\begin{array}{cc}
\mathbf{R}_{11} & \mathbf{R}_{12} \\
\mathbf{0}_{4} & \mathbf{R}_{22}
\end{array}\right],
$$

where $\mathbf{R}_{11}$ and $\mathbf{R}_{22}$ are diagonal matrices, and $\mathbf{0}_{4}$ is a $4 \times 4$ zero matrix. This interesting property comes from the special structure of the real generator matrix $\mathbf{G}$. Indeed, one can show that the real generator matrix of (7) can be decomposed as

$$
\mathbf{G}=\left[\begin{array}{ll}
\mathbf{G}_{1} & \mathbf{G}_{2}
\end{array}\right],
$$

where each $\mathbf{G}_{i}$ is equivalent to the real generator matrix of the Alamouti scheme. This allows us to decouple the estimation of symbol pairs and simplify the receiver architecture. More formally, this allows the SD to be performed only for 4 levels and the SD finds

$$
\hat{\mathbf{s}}_{R}^{(5,8)}=\underset{\overline{\mathbf{s}}_{R}^{(5,8)}}{\arg \min }\left\|\overline{\mathbf{y}}_{R}^{(5,8)}-\mathbf{R}_{22} \overline{\mathbf{s}}_{R}^{(5,8)}\right\|^{2} .
$$

Here, we used the notation $\mathbf{x}^{(k, l)}=\left[x_{k}, \ldots, x_{l}\right]^{T}$ in which the symbols are collected from the vector $\mathbf{x}$ either in increasing or decreasing order of indices from $k$ to $l$. Once the symbol vector $\overline{\mathbf{s}}_{R}^{(5,8)}$ is obtained using the reduced SD, the remaining symbols collected in $\overline{\mathbf{s}}_{R}^{(1,4)}$ are decoded simply as in the case of Alamouti scheme using symbol-by-symbol 'Alamouti' decoding. Moreover, since the matrix $\mathbf{G}$ is a combination of two 'Alamouti-type' real generator matrices, the decoding can be performed the other way round with the same complexity: By decoding in the reverse direction, the SD finds

$$
\hat{\overline{\mathbf{s}}}_{R}^{(4,1)}=\underset{\overline{\mathbf{s}}_{R}^{(4,1)}}{\arg \min }\left\|\overline{\mathbf{y}}_{R}^{(4,1)}-\mathbf{R}_{11} \overline{\mathbf{s}}_{R}^{(4,1)}\right\|^{2},
$$

and the remaining symbols in $\overline{\mathbf{s}}_{R}^{(8,5)}$ are obtained using symbol-by-symbol 'Alamouti' decoding. This simply allows evaluating soft log-likelihood ratios for all symbol bits with the same receiver architecture. This is particularly important if we need soft data for further decoding stages - which is the case in real system architectures.

It is also worth noting that since the matrix $\mathbf{R}_{22}$ (resp., $\mathbf{R}_{11}$, for the reverse detection order) is diagonal, the number of computation will be reduced in the SD process in (22) (resp. in (23)) compared to the standard SD computations with 4 level tree search. 


\section{NUMERICAL COMPARISONS}

We now provide some performance comparisons between the aforementioned STCs in order to sustain our claims. Fig. 1 shows the BER performance as a function of $E_{b} / N_{0}, E_{b}$ denoting the average signal energy per bit, and provides comparisons between $\mathbf{X}_{\text {new }}$, namely, the new STC, and $\mathbf{X}_{C}$ (Matrix C) of WiMAX systems. The results are obtained for an uncorrelated Rayleigh fading channel with $E\left[\left|h_{k l}\right|^{2}\right]=1$ for all $k, l$, using full SD for $\mathbf{X}_{C}$ and reduced complexity SD (cf. Section IV.B) for $\mathbf{X}_{n e w}$. It can be seen that $\mathbf{X}_{\text {new }}$ gives essentially the same results as $\mathbf{X}_{C}$ at substantially lower complexity. The small difference between the curves in Fig. 1 can be interpreted as the performance loss due to the receiver complexity reduction. The performance curves for the STC proposed in [7] were not included for readability purposes. As the coding gains are very close to each other, intuitively, both are expected to give very close results. Indeed, we have observed that the performance curves of $\mathbf{X}_{\text {new }}$ and the one proposed in [7] almost fits on each other. Such comparisons also exist in [13] and coincide with our observations.

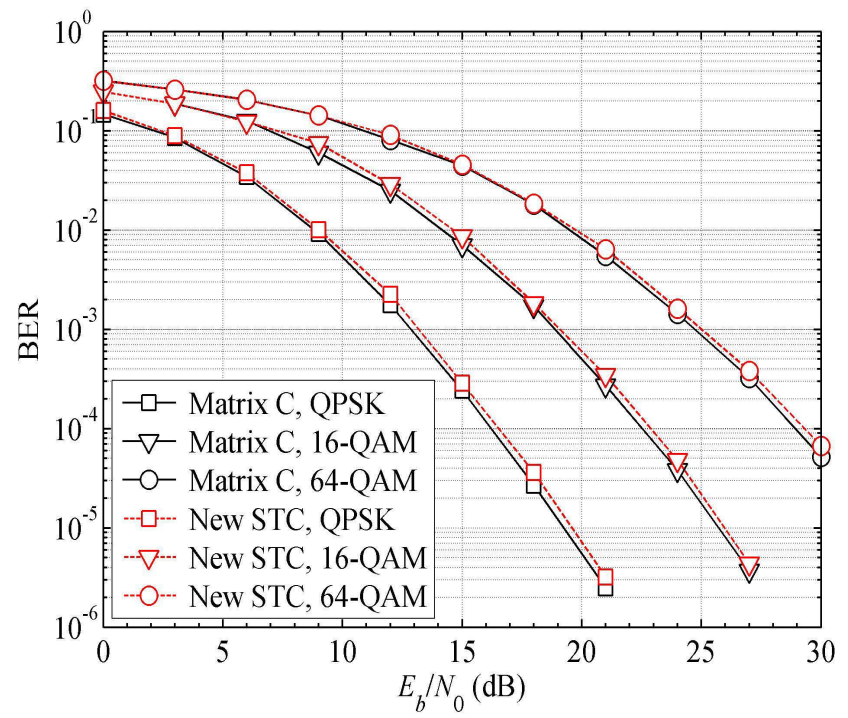

Fig. 1: Performance comparison between $\mathbf{X}_{C}$ with full SD and $\mathbf{X}_{\text {new }}$ with reduced SD.

The complexity reduction can be observed from Fig. 2, where the number of visited nodes [18] are plotted as a function of SNR $E_{b} / N_{0}$. As seen in Fig. 2, the new STC results in a considerable reduction in the number of computations. Moreover, since the number of visited nodes has a large impact on the required chip area per throughput [18], the new STC reduces the hardware complexity without any noticeable performance degradation.

We shall now compare the performance of $\mathbf{X}_{n e w}$ with that of $\mathbf{X}_{C}$ when the same suboptimum receiver is used for both. In order to observe such a comparison, for both $\mathbf{X}_{C}$ and $\mathbf{X}_{n e w}$, we initially use SDs with tree search levels of 2, 4 and 6, and then employ zero-forcing decision-feedback equalization (ZF-DFE) for the rest of the symbols. In Fig. 3, we depict the performance curves for QPSK where we employed ZF-DFE for detecting 2, 4 and 6 real symbols, respectively. Notice that ZF-DFE of 2 and 4 symbols corresponds to the optimum detection for $\mathbf{X}_{n e w}$. For similar detection complexities, $\mathbf{X}_{\text {new }}$ outperforms $\mathbf{X}_{C}$ about $2.4 \mathrm{~dB}$ at the BER of $10^{-3}$ when a 2stage ZF-DFE is used and this gain increases to about $8.1 \mathrm{~dB}$ when a 4-stage ZF-DFE is used. On the other hand, when we use SD only for the first two real symbols and detect the rest using ZF-DFE, neither of the two STCs benefits from the available diversity. For low SNR values, $\mathbf{X}_{\text {new }}$ provides better performance than $\mathbf{X}_{C}$, while for high SNR values $\mathbf{X}_{C}$ slightly outperforms $\mathbf{X}_{\text {new }}$. Similar conclusions can be drawn for 16QAM as depicted in Fig. 4.

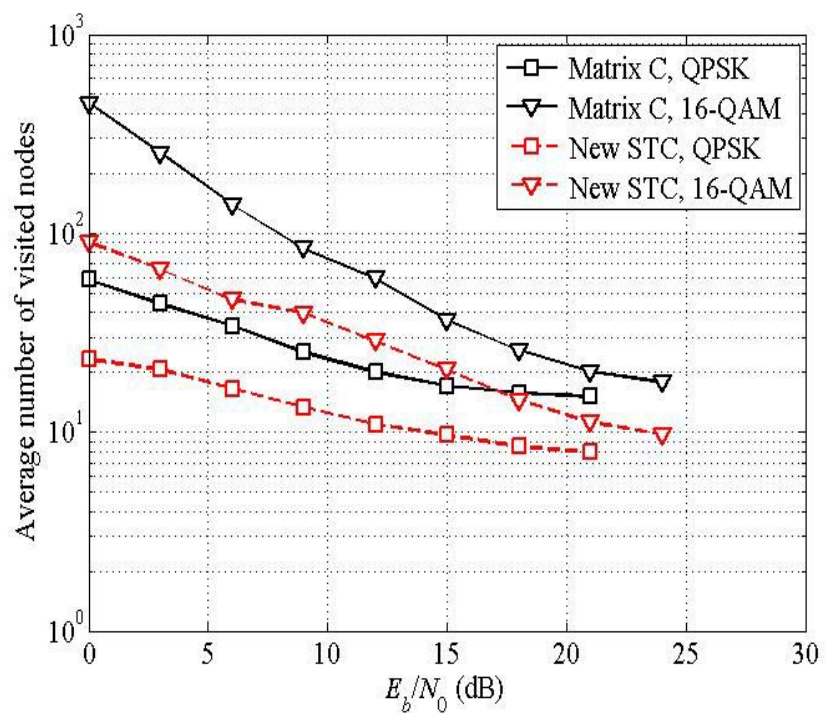

Fig. 2: Average number of visited nodes in full SD used to decode $\mathbf{X}_{C}$ and reduced SD used to decode $\mathbf{X}_{\text {new }}$.

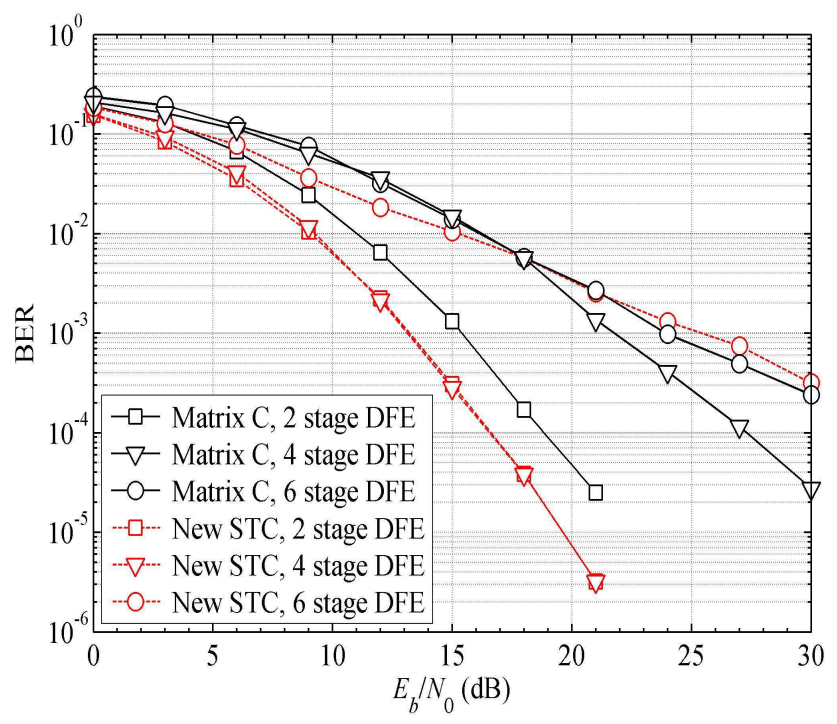

Fig. 3: Performance comparison between $\mathbf{X}_{C}$ and $\mathbf{X}_{n e w}$ with the same detector complexity (QPSK). 


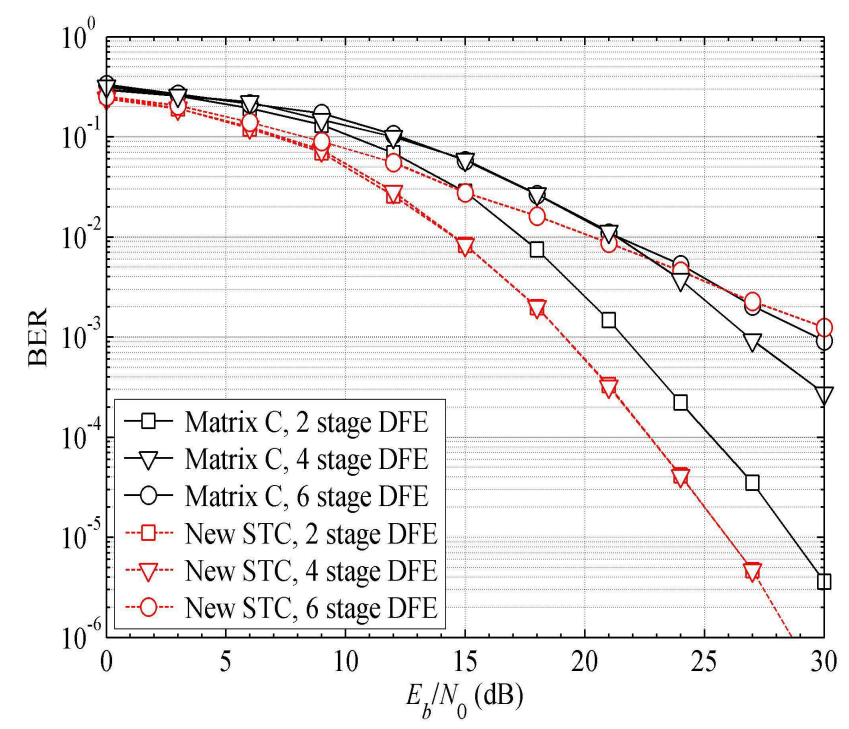

Fig. 4: Performance comparison between $\mathbf{X}_{C}$ and $\mathbf{X}_{n e w}$ with the same detector complexity (16-QAM).

\section{CONCLUSIONS}

In this paper, we have analyzed the MIMO schemes of IEEE 802.16 specifications and made a comparison between the existing FR-FD $2 \times 2$ STCs and other possible candidates. The results indicated that the recently proposed schemes achieve the performance of the existing STC, while substantially reducing the optimum decoder complexity. Furthermore, it was also observed that when used at similar decoder complexity, the new STCs may bring a considerable performance gain compared to the existing STCs. Thus, the new STC designs with reduced decoder complexity open up new perspectives for future evolutions of WiMAX systems, as well as for other broadband wireless systems.

\section{ACKNOWLEDGEMENTS}

The authors S. Sezginer and H. Sari would like to acknowledge the support of the European Commission through the FP7 project WiMAGIC (see www.wimagic.eu).

\section{REFERENCES}

[1] IEEE 802.16-2005: IEEE Standard for Local and Metropolitan Area Networks - Part 16: Air Interface for Fixed and Mobile Broadband Wireless Access Systems - Amendment 2: Physical Layer and Medium Access Control Layers for Combined Fixed and Mobile Operation in Licensed Bands, Feb. 2006.

[2] S. M. Alamouti, "A simple transmit diversity technique for wireless communications," IEEE J. Sel. Areas Commun., vol. 16, pp. 1451-1458, Oct. 1998.

[3] J.-C. Belfiore, G. Rekaya, and E. Viterbo, "The Golden code: A $2 \times 2$ full-rate space-time code with non-vanishing determinants," IEEE Trans. Inf. Theory, vol. 51, pp. 1432-1436, Apr. 2005.

[4] H. Yao and G. W. Wornell, "Achieving the full MIMO diversitymultiplexing frontier with rotation-based space-time codes," in Proc. Allerton Conf. Commun., Control, and Computing, Monticello, IL, Oct. 2003.

[5] P. Dayal and M. K. Varanasi, "An optimal two transmit antenna spacetime code and its stacked extensions," IEEE Trans. Inf. Theory, vol. 51 no. 12, pp. 4348-4355, Dec. 2005.

[6] S. Sezginer and H. Sari, "Full-rate full-diversity $2 \times 2$ space-time codes of reduced decoder complexity," IEEE Commun. Lett., vol. 11, no. 12, pp. 973-975, Dec. 2007.

[7] J. Paredes, A.B. Gershman, and M. G. Alkhanari, "A $2 \times 2$ space-time code with non-vanishing determinants and fast maximum likelihood decoding," in Proc. ICASSP'07, Honolulu, Hawaii, USA, Apr. 2007.

[8] J-C. Guey, M.P. Fitz, M.R. Bell, and W-Y. Kuo, "Signal design for transmitter diversity wireless communications systems over Rayleigh fading channels," in Proc. VTC'96 Spring, 1996, pp. 136-140.

[9] F. Oggier and E. Viterbo, "Algebraic number theory and code design for Rayleigh fading channels," in Foundations and Trends in Commun. and Inf. Theory, vol. 1, pp. 333-415, 2004.

[10] V. Tarokh, N. Seshadri, and A. R. Calderbank, "Space-time codes for high data rate wireless communications: Performance criterion and code construction," IEEE Trans. Inf. Theory, vol. 44, pp. 744-765, Mar. 1998.

[11] M. Samuel and M. P. Fitz, "Reducing the detection complexity by using $2 \times 2$ multi-strata space-time codes," in Proc ISIT'07, Nice, France, June 2007, pp. $1946-1950$.

[12] O. Tirkkonen and R. Kashaev, "Combined information and performance optimization of linear MIMO modulations," in Proc ISIT'02, Lausanne, Switzerland, June 2002, p. 76.

[13] E. Biglieri, Y. Hong, and E. Viterbo, "On fast-decodable space-time block codes," submitted for publication, 2007 (available in arXiv:0708.2804v1 [cs.IT] 21 Aug 2007)

[14] P. Elia et al., "Explicit construction of space-time block codes: Achieving the diversity-multiplexing gain tradeoff," IEEE Trans. Inf. Theory, vol. 52, pp. 3869-3884, Sep. 2006.

[15] B. Hassibi and B. Hochwald, "High-rate codes that are linear in space and time," IEEE Trans. Inf. Theory, vol. 48, pp. 1804-1824, July 2002.

[16] M. Gharavi-Alkhansari and A. B. Gershman, "Constellation space invariance of orthogonal space-time block codes," IEEE Trans. Inf. Theory, vol. 51, pp. 1051-1055, Jan. 2005.

[17] E. Viterbo and J. Boutros, "A universal lattice code decoder for fading channels," IEEE Trans. Inf. Theory, vol. 45, pp. 1639-1642, Jul. 1999.

[18] A. Burg et al., "VLSI implementation of MIMO detection using sphere decoder algorithm," IEEE J. Solid-State Circuits, vol. 40, pp. 15661577 , July 2005 